\title{
Treatment outcomes in depression: reducing drop-out rates in cognitive therapy
}

\author{
Alexandra D. Pentaraki
}

\begin{abstract}
SUMMARY
Although cognitive therapy is a promising treatment for depression, high drop-out rates and, conversely, the specific elements that make the intervention effective continue to puzzle practising clinicians. This article discusses both therapistrelated factors (including competence and competencies) and patient-related factors (such as engagement in therapy and external logistical problems with attendance) that are related to drop-out and suggests practical ways to address them and improve outcomes. It examines cognitive and behavioural elements of manualised cognitive therapy and discusses findings relating to cognitive-behavioural therapy (CBT) in particular.
\end{abstract}

\section{LEARNING OBJECTIVES}

- Understand factors associated with high dropout rates in the cognitive therapy of depression and ways to reduce them

- Learn about treatment integrity in cognitive therapy of depression and its relationship to the clinical outcome

- Identify specific cognitive therapy techniques associated with patients' improvement in depression

\section{DECLARATION OF INTEREST}

None.

Depression, or major depressive disorder (MDD), is defined in DSM-5 as an illness that affects mood, thinking and behaviour and it is characterised by feelings of sadness and loss of interest in previously pleasurable activities (American Psychiatric Association 2013). It is a potentially chronic condition and DSM-5 includes new diagnostic conceptualisations of the illness: the new diagnostic criteria for depressive disorders assume that most individuals with chronic depression or MDD will fulfil the criteria for dysthymia, so chronic depression and dysthymia have been merged into the diagnostic category of persistent depressive disorders (PDD). Nevertheless, not all individuals with chronic depression/MDD will meet the diagnostic criteria for PDD (American Psychiatric Association 2013; Uher 2014).

People who have experienced MDD have a high risk of relapse during the first 6 months after symptom remission. Those who have been prescribed antidepressant medication are therefore advised to continue the antidepressant for at least 6 months after remission. In a study comparing relapse rates following successful treatment with medication or cognitive therapy, Hollon et al (2005) found that $70 \%$ of those who had responded to antidepressants relapsed within 12 months of medication withdrawal, whereas only $31 \%$ of responders to cognitive therapy relapsed during that period. The authors thus concluded that cognitive therapy had more enduring results than antidepressants in MDD treatment and relapse prevention. A metaanalysis by Cuijpers et al (2013) that compared the treatment efficacy of psychotherapies and pharmacotherapy found that cognitive-behavioural therapy (CBT) plus medication was slightly better than CBT alone. This suggests the need for more studies to clarify the effects of addition of antidepressants to cognitive therapy and CBT.

\section{Cognitive therapy for depression}

Cognitive therapy as a component of CBT uses cognitive restructuring techniques to change the individual's negative and maladaptive thinking. Cognitive therapy asserts that the way people perceive and think about themselves, others and the world influences their feelings and behaviour (including physiological reactions). The cognitive theory of depression conceptualises it as a psychological mechanism characterised by increased negative thinking about oneself, the world and the future (the 'depressive cognitive triad'), negative core beliefs, cognitive errors, increased hopelessness and mood-congruent recall (Beck 1979, 2009; Haaga 1991; Westbrook 2007; Pentaraki 2012). Cognitive therapy asserts that, by challenging and changing negative and maladaptive thoughts, the negative feelings that are prevalent in depression will diminish and the person's functioning will improve.
Alexandra D. Pentaraki is a clinical psychologist and neuropsychologist in private practice with a clinica and research interest in depression, anxiety, trauma and psychosis. She is also a registered expert witness in European courts. From 2012-2016 she was an honorary lecturer in Applied Psychology \& Mental Health at the University of Liverpool/ Laureate Education, where she taught, researched and supervised research. She is an honorary visiting research associate at the Institute of Psychiatry, Psychology and Neuroscience (loPPN), King's College London, a Chartered Psychologist and an Associate Fellow of the British Psychological Society. Correspondence Dr Alexandra Pentaraki, Brain Matters Institute, Tsimiski 10, 54624, Thessaloniki, Greece. Email: brainmattersinstitute@windowslive.com

\section{Copyright and usage} (C) The Royal College of Psychiatrists 2018 
Cognitive therapy is one of the most manualised psychological treatments, although recently the third-wave of CBT has included other therapeutic modalities such as the mindfulness and compassionate therapy. The combination of cognitive therapy with mindfulness is considered to be effective in relapse prevention in $\mathrm{MMD}$, with evidence that it can reduce further relapse by $55 \%$ in people who have experienced three or more depressive episodes (Teasdale 2000; Ma 2004). The National Institute for Health and Care Excellence (NICE) has proposed mindfulness-based cognitive therapy as the treatment of choice for the management of depression in people at significant risk of relapse, those who have residual symptoms and for those who are currently well but have had three or more previous episodes (National Institute for Health and Care Excellence 2009: para. 1.9).

A study of temporal trends in the reported efficacy of manualised CBT as a treatment for depression showed a decline in its effects since its introduction in the 1970s. One factor found to be associated with less effective CBT was the therapist's experience: patients who received CBT from experienced psychologists showed a more significant reduction in their depressive symptoms than those who received CBT from psychology students with less experience in therapy while another factor was associated with deviations from using the manual or lack of adhering to the manual (Johnsen 2015). Nevertheless, the results of the study must be interpreted with caution owing to its methodological limitations (Waltman 2016).

\section{Drop-out rates}

Drop-out rates are very high for all forms of psychotherapy and the reasons for this are still under investigation. A meta-analysis of 125 studies reported a mean drop-out rate of almost 47\%. However, rates differed significantly as a function of the definition of drop-out. Lower rates were recorded when it was defined as termination of therapy because of failure to attend a scheduled session, rather than by either the number of sessions attended or the therapist's judgement. The study reported significant effect sizes for three patient demographic variables: 'racial status', education and income. Specifically, African Americans and other minority groups, the less educated and groups with lower income showed higher drop-out rates (Wierzbicki 1993). Similar results were also reported in recent studies (Arnow 2007).

Relatively few studies have examined the effects of ethnicity or culture on drop-out rates in CBT for depression and those that do lack a comparison with other types of treatment. Emerging evidence shows that CBT can be effective for depression in patients from ethnic minority groups. In a review of the literature, Horrell (2008) reported its efficacy for patients in the USA from ethnic minority backgrounds such as African American and Hispanic/Latino, with 7 out of 12 studies showing significant reduction of depressive symptoms compared with placebo or waiting-list control. Bennett et al (2014) reported a reduction of symptom severity in a group of Maori patients with mild to severe depression who received a culturally adapted CBT. In a randomised controlled trial (RCT), Naeem et al (2015) reported a significant improvement of depressive symptoms in Pakistani out-patients with depression who received a culturally adapted CBT plus standard treatment in comparison with out-patients who received standard treatment only. However, it is clear that we need more studies in order to understand the effect of ethnicity on dropout rates, particularly RCTs that include a comparison between culturally modified and non-culturally modified CBT in ethnic minority patients.

Drop-out rates, particularly for premature termination of psychotherapy, do not seem to be associated with type of psychotherapeutic approach or the particular disorder, with the exception of depression, post-traumatic stress disorder (PTSD) and eating disorders. A meta-analysis by Swift \& Greenberg (2014) compared drop-out rates between different psychotherapeutic approaches for 12 separate disorder categories. It reported that integrative treatments were related to the lowest average drop-out rates for depression and PTSD, and dialectical behaviour therapy resulted in the lowest average drop-out rate for eating disorders; treatments did not differ in their drop-out rates for disorders such as bereavement, borderline personality disorder, generalised anxiety disorder, obsessive-compulsive disorder, other personality disorders, panic disorder, psychotic disorders, social phobia and somatoform disorders. The findings for depression suggest that it is the therapist's understanding of which approach or set of approaches might work for each individual patient that is important in retaining them in therapy. The non-significant difference in drop-out rates for the remaining disorders may suggest that the patient's decision to drop out may depend on common factors (e.g. the therapeutic alliance, having a rationale for the problem and a believed-in method for treating it) and patient and therapist characteristics. These results make it essential for clinicians to understand drop-out rates in cognitive therapy.

Methodological difficulties in studying drop-out rates in psychotherapy, and in cognitive therapy in particular, include the definition of drop-out, as no standard definition has been used. Some 
authors define drop-out as ending the treatment before achieving the proposed targets of therapy or stopping therapy against the therapist's advice, whereas others define it in terms of failure to attend a scheduled session and not subsequently returning. A study that examined drop-out rates from CBT among 203 patients, most of whom had anxiety and affective disorders, reported that most drop-outs happened in the early stages of therapy (by about the 14th session). Of the total who dropped out (43.8\%), $46.7 \%$ gave the reason as low motivation and/or dissatisfaction with the therapist or treatment, $40 \%$ said that there were external difficulties (e.g. transportation problems, timetables, new responsibilities) and $13.3 \%$ said that they felt that they had improved. Individual differences such as age, gender, the duration of the disorder, use of medication and employment status did not affect drop-out rates (Bados 2007).

A large study of over 10000 participants receiving various psychological interventions addressed the association between the number of treatment sessions and clinically significant improvement. Analyses of dose-response data showed that 50\% of patients required 21 sessions of treatment in order to reach clinically significant improvement; $75 \%$ of patients were predicted to improve only after receiving more than 40 treatment sessions (Lambert 2001). However, it is important to note that in many cases patients improve with fewer than 20 sessions and this fact must be considered when we define drop-out rates in psychotherapy and cognitive therapy.

\section{Treatment integrity: therapist adherence and competence}

The study of treatment integrity involves the assessment of two constructs: adherence and competence. Adherence refers to the extent to which a therapist uses the interventions and approaches specified in the manual and avoids any that are not specified, whereas competence is concerned with the therapist's skill in delivering the treatment (Waltz 1993). Therapist competence is not clearly defined in the literature and there are different conceptualisations of what exactly it entails (for instance, different variables may be included, such as non-specific factors, supervisory experiences, level of training, the clinical context), making competence more complex to study than adherence (McGlinchey 2003).

In a review of the literature on treatment integrity in cognitive therapy for depression, McGlinchey $\&$ Dobson (2003) noted that the study of adherence and competence overlaps, although competence presumes adherence but adherence does not presume competence. The review reported that studies that examined the relationship between adherence to protocol and outcome in cognitive therapy showed that the variable labelled 'CT-concrete', reflecting pragmatic aspects of the intervention such as the establishment of a session's agenda and homework assignment, positively correlated with improvement, whereas the variable 'CT-abstract', reflecting the therapist's exploration of underlying assumptions and personal meanings, did not correlate with outcome. This suggests that specific elements of cognitive therapy and not the whole cognitive therapy protocol are related to an effective outcome.

Similarly, a study that compared component techniques of CBT (behavioural activation and modification of automatic thoughts) and full CBT in depression showed that, although there was excellent adherence by the therapists to all three treatment protocols, full CBT did not produce better outcomes at the end of acute treatment or at 6-month follow-up than behavioural activation or modification of automatic thoughts. This suggests that both behavioural activation and modification of automatic thoughts are as effective as full CBT at changing negative thinking and dysfunctional attribution styles in depression (Jacobson 1996).

A three-centre study of CBT for depression showed that therapist competence, the second ingredient of treatment integrity, did not correlate significantly with outcome, specifically with the reduction of patients' depressive symptoms. However, it did find that the component of competence that was most related to outcome was the factor that reflected the therapist's ability to structure the treatment (Shaw 1999).

\section{Comorbid psychiatric disorders}

Comorbidity of depression with other disorders is another factor that influences clinical outcome. Cognitive therapy seems to be a promising approach in the treatment of depression and comorbid anxiety or personality disorders, but drop-out from cognitive therapy has not been studied among this group of patients. Anxiety is commonly comorbid with depression and it is related to greater symptom severity and dysfunction (Fava 2004). Forand \& DeRubeis (2013) reported that CBT was not effective in the treatment of depressive symptoms in patients with moderate to severe depression and comorbid anxiety disorders. However, the study found that patients with higher pre-treatment anxiety levels showed an early rapid improvement of depressive symptoms with both CBT and medication, but the improvement was not sustained. Personality disorders are also commonly comorbid with MDD. They predict the persistence of 
depressive symptoms and reduce rates of treatment response in psychotherapy (Newton-Howes 2014). In the cognitive therapy of MDD and comorbid personality disorder, it is important to target the symptoms of the personality disorder: if the personality disorder symptoms persist after remission of depressive symptoms, the patient is at greater risk of relapse of depression (Beck 2015). Keefe et al (2016) reported that the early use of cognitive techniques targeting maladaptive core beliefs ('CTbelief' techniques) predicted significant improvements in depressive and personality disorder symptoms (compared with depressive symptoms in patients without personality disorder), suggesting that the early focus on patients' core beliefs may be efficacious in the treatment of MDD and comorbid personality disorder.

\section{Reducing drop-out rates and improving outcomes}

\section{Completed homework/action plans}

Cognitive therapy and CBT are characterised by a collaborative relationship that involves the active participation of the patient and their engagement between sessions in 'homework' (also known as 'action plans') (Beck 2016). Studies suggest a positive association between homework and therapeutic outcome. A meta-analysis of 27 studies $(N=1702)$ of CBT with and without homework assignments for a range of disorders showed the efficacy (with medium effect sizes) of homework on the therapeutic outcome (Kazantzis 2000).

This efficacy of homework assignments also seems to be evident in people with depressive and anxiety disorders. Rees et al (2005) reported that the amount of completed homework (thought diaries, reading tasks, relaxation exercises, behavioural tasks such as exposure and pleasurable activities) predicted the clinical outcome of patients with depression and anxiety. The levels of pre-treatment symptoms of depression and anxiety were not related to homework tasks such as completed relaxation exercises, readings tasks, thought diaries and behavioural tasks. The findings suggest that: (a) the completion of homework in CBT is associated with the clinical outcome (reduction of symptoms) independently of the severity of pre-treatment symptoms of depression and anxiety; (b) there is a relationship between the type of homework completed and symptom change: for example, behavioural tasks (both exposure and pleasurable activities) are more effective in reducing depression, whereas the completion of thought diaries is associated with reduced anxiety.

The integration of these findings into clinical practice will help clinicians to design homework that meets the needs of individual patients (e.g. what would be a pleasurable activity for this particular person?). Also, the findings seem to validate the everyday clinical observation that one of the core symptoms of depression, and also a maintenance factor, is the lack of pleasure. Therapy can help the patient to understand this and to change it by seeking pleasure not only in activities, but also in people who are understanding and supportive. The patient can be helped to identify pleasurable activities by recording their daily activities and the emotions associated with them. This is the first step in preparing for 'activity scheduling'. After a week or two of this monitoring, the individual can understand which activities are pleasurable and which no longer give them pleasure or contribute to their negative mood. In collaboration with their therapist they can prepare an activity schedule that substitutes non-pleasurable activities with pleasurable ones.

\section{Therapist flexibility and patient outcome}

A major criticism of cognitive therapy and CBT is that they lack flexibility, owing to the structured way the therapy is conducted. Different patients have different needs and this must be taken into consideration. The establishment of a good therapeutic alliance, the bedrock of collaborative empiricism, is very important during the first stages of therapy. Patients may face difficulties with, for example, trust or confidentiality and these need to be addressed before any therapeutic technique is introduced. Nevertheless, clinical practice shows that, even in the first session, patients can be introduced to basic aspects of cognitive therapy in relation to their depression, thus allowing the therapist to be more flexible with the treatment protocol. An early introduction to cognitive therapy (or CBT) and its process can help patients to see their active role in therapy and will enhance its collaborative aspect.

\section{Therapist competence and competencies}

Therapist competence and competencies (confusingly, also known as therapist competency and competences) are associated with successful therapeutic outcomes in all types of psychotherapy, including empirically supported treatments (ESTs) such as cognitive therapy and CBT. The therapist's competence refers to their overall capacity to function as a professional psychologist/ clinician, i.e. how competent they are. The therapist's competencies are the specific abilities they have, such as the ability to engage with a patient, their knowledge of particular psychological disorders and the ability to intervene appropriately in therapy (Kaslow 2004). Competencies for ESTs 
such as cognitive therapy and CBT for depression and anxiety fall into two dimensions: the first dimension involves general competencies required for conducting psychotherapy (generic therapeutic competencies) and the second involves competencies necessary for the implementation of the specific mode of treatment (for example, CBTrelated domains). Box 1 gives examples of both dimensions.

Therapist competence is associated with outcome in cognitive therapy for depression. Trepka et al (2004) used the Cognitive Therapy Scale (CTS) to rate a randomly selected therapy session from each of 30 sessions of cognitive therapy for depression. They found that both therapeutic alliance and therapist's competence were related to outcome. The therapeutic alliance was significantly related to outcome when controlling for the effect of competence but not vice versa, suggesting the important effect of the therapist's competence on the therapeutic relationship and on the reduction of

BOX 1 Competencies for an empirically supported treatment such as cognitivebehavioural therapy (CBT) for depression and anxiety

General (generic) therapeutic competencies

- Knowledge and understanding of mental disorders

- Working within ethical guidelines

- Ability to develop a good therapeutic relationship with the patient

- Conducting a generic assessment

CBT-related domains

- Basic CBT competencies:

- setting an agenda for each session

- agreeing with the patient the goals of the interventions

- Specific CBT techniques:

$\circ$ thought diaries

$\circ$ activity scheduling

- guided discovery

- exposure techniques

- Problem-specific competencies:

$\circ$ self-help interventions

$\circ$ more intense psychotherapy

- Meta-competencies:

$\circ$ ability to adapt the processes and techniques of CBT to the needs of the patient

- ability to implement therapy appropriately

(Roth 2008) depression, especially in patients who completed therapy. Items measured on the CTS included not only the therapist's skill, but also patient factors such as the patient's ability to engage in the tasks of cognitive therapy. The findings suggest that both generic therapeutic competencies (e.g. the formation of a therapeutic alliance) and therapy-specific competencies such as expertise and skills in CBT techniques are significant factors in the successful treatment of depression, and this should be taken into consideration in both course design and clinical supervision. Therapists themselves need to find ways, through the development of a good therapeutic relationship with the patient, to persuade them to complete therapy.

Similar results that support the significance of therapists' competence, in terms of their experience with cognitive therapy, in the treatment of depression come from a multi-site study by DeRubeis et al (2008). This showed that improvement in moderate to severe depression was significantly associated with the availability of experienced cognitive therapists: treatment by less experienced cognitive therapists was related to non-significant improvement.

\section{Clinical supervision}

Clinical supervision has been suggested to have a positive effect on clinical outcomes, but the results of published studies are not consistent: for example, Bambling et al (2006) reported a positive effect on patient outcomes, whereas White $\mathcal{E}$ Winstanley (2010) reported no significant effects. One of the few studies to have examined the effect of clinical supervision on therapists' delivery of CBT to patients with depression found that the therapists' gender and their anxiety levels both influenced the supervisors' focus in supervision, suggesting that supervisors show biases in the way they support the work of different clinicians (Simpson-Southward 2016). Thus, there is a need to increase our understanding of the potential effect of clinical supervision on treatment effectiveness and drop-out rates in cognitive therapy and CBT.

\section{Specific cognitive therapy techniques}

Cognitive therapy techniques and their association with clinical outcome and drop-out rates in depression have not been studied extensively. However, there is some emerging evidence that shows that specific techniques are positively associated with relapse prevention. An RCT that examined the use of cognitive therapy following remission to prevent subsequent relapse in recurrent depression reported that certain cognitive techniques were associated 
with fewer relapses/recurrences of depression, especially in patients who had experienced five or more previous depressive episodes (Bockting 2005). The techniques concerned were the identification of negative thoughts and dysfunctional attitudes using self-report questionnaires and the vertical arrow technique, and their change through Socratic questioning and the identification of positive attitudes. Although the study shows the potential protective effects of cognitive therapy in preventing the recurrence of depression, the results should be interpreted with caution as the study did not control for the effect of continuing medication for some participants (the use of pharmacotherapy did not preclude entry to follow-up).

Behavioural activation, a component of CBT, is also associated with effective outcomes in depression, irrespective of illness severity, and in relapse prevention. Behavioural activation focuses primarily on activity scheduling to encourage patients to approach activities that they are avoiding and on functional analysis of the cognitive processes (such as rumination) that promote avoidance (Martell 2001; Veale 2008). An RCT that compared behavioural activation, standard CBT, antidepressant medication (paroxetine) and a drug placebo in 214 out-patients with MDD found that behavioural activation was comparable to paroxetine, and the combination of behavioural activation with paroxetine was more efficacious than CBT alone (Dimidjian 2006). Behavioural activation was associated with a greater percentage of participants achieving remission.

Behavioural activation alone seems to be a costeffective treatment for MDD as it can be easily taught to junior mental health workers in primary care, but it is not superior to $\mathrm{CBT}$ in reducing depressive symptoms. An RCT by Richards et al (2016) reported that the use of behavioural activation in a primary care clinical setting and in psychological therapy services was cost-effective and affordable compared with CBT.

It is interesting to note that behavioural activation conceptualises and treats the negative thinking that characterises depression differently than cognitive therapy. Behavioural activation is interested in the function of negative and ruminative thinking, such as the consequences that it creates (e.g. avoidance, withdrawal), and the implementation of activation strategies, whereas cognitive therapy is interested in the content of thought. Also, in behavioural activation, ruminative thinking is countered by directing attention to sensations, thus shifting it away from the content of the thoughts. The great emphasis on the utility of thought in behavioural activation may contribute to its effectiveness as a treatment for depression, but this remains to be explored in further studies.

Finally, although the changing of negative thoughts in cognitive therapy has been associated with effective treatment of depression, the challenging of maladaptive thoughts has not been supported by all studies. In a critical review of the literature that examined the effect of cognitive mediation (challenging maladaptive thoughts) in CBT for depression and anxiety, Longmore $\&$ Worrell (2007) found little evidence that supports a significant effect of cognitive mediation on symptom reduction. However, more studies are needed to support this claim.

TABLE 1 Selection of studies that show the efficacy of specific therapeutic techniques of manualised cognitive therapy and cognitive-behavioural therapy (CBT) in the treatment of depression

\begin{tabular}{|c|c|}
\hline Studies & Techniques \\
\hline McGlinchey \& Dobson (2003) & $\begin{array}{l}\text { 'CT-concrete' (reflecting pragmatic aspects of cognitive therapy such as the establishment of a } \\
\text { session's agenda and agreeing homework assignments with the patient) }\end{array}$ \\
\hline Jacobson et al (1996) & Behavioural activation and modification of automatic thoughts \\
\hline $\begin{array}{l}\text { Kazantzis et al (2000); Rees et al } \\
\text { (2005) }\end{array}$ & $\begin{array}{l}\text { Completed homework/action plan, specifically: exposure and pleasurable activities for the } \\
\text { reduction of depression; thought diaries for the reduction of anxiety }\end{array}$ \\
\hline Trepka et al (2004) & $\begin{array}{l}\text { Therapist's competence (e.g. expertise and skills in CBT techniques) and generic therapeutic } \\
\text { competencies (e.g. the formation of a therapeutic alliance) }\end{array}$ \\
\hline $\begin{array}{l}\text { DeRubeis et al (2008); Johnsen \& } \\
\quad \text { Friborg (2015) }\end{array}$ & Therapist's experience in cognitive therapy \\
\hline $\begin{array}{l}\text { Bockting et al (2005) (on relapse } \\
\text { prevention) }\end{array}$ & $\begin{array}{l}\text { Identification of negative thoughts and dysfunctional attitudes using self-report questionnaires and } \\
\text { the vertical arrow technique; changing negative thoughts through Socratic questioning and } \\
\text { identification of positive attitudes }\end{array}$ \\
\hline Dimidjian et al (2006) & Behavioural activation and antidepressant medication \\
\hline
\end{tabular}




\section{Conclusions}

Manualised cognitive therapy is a promising treatment for depression, regardless of symptom severity, and it is also efficacious in relapse prevention. Table 1 summarises studies that show the efficacy of specific therapeutic techniques of manualised cognitive therapy and CBT in the treatment of depression. Higher drop-out rates are observed during the first stages of treatment and they seem to be related to the patient's low motivation, dissatisfaction with the therapist or treatment, and external difficulties. Cognitive therapists can tackle these issues by monitoring the patient's behaviour and thoughts about therapy, especially during its early stages. The therapist's flexibility in explaining the aims of the therapeutic work, the meaning of action plans (homework assignments) and the collaborative nature of cognitive therapy will help patients to gain an understanding of therapy and introduce the feeling that they are not alone in this effort.

The cognitive therapist needs to consider CT-concrete variables that have a positive effect on the patients' progress, such as the establishment of each session's agenda and homework assignments/ action plans. The therapist's ability to structure the treatment is associated with reduction of the patient's depressive symptoms, so therapists must be able to provide a well-designed and structured treatment. The introduction of action plans correlates significantly with therapeutic outcome in CBT. Therapists should be aware that specific action plans are associated with the reduction of depressive and anxious symptoms independently of pre-treatment levels of depression and anxiety. Therapists should be proactive in negotiating with the patient and persuading them to complete homework/action plans. Action plans that include both exposure and pleasurable activities are more effective in reducing depression, whereas the completion of thought diaries seems to be associated with reduced anxiety. The use of activity scheduling can help therapists in the design of successful action plans.

Cognitive therapists should seek, via training and supervision, to gain competencies in generic therapeutic skills (e.g. the formation of a good therapeutic alliance) and expertise in cognitive therapy techniques and skills. Both generic therapeutic skills and competence in cognitive therapy techniques are related to a good therapeutic outcome in depression, and thus training in cognitive therapy needs to include these aspects in its modules and professional development. Specific cognitive therapy techniques have been associated with effective outcomes and relapse prevention in depression. Therapists should be able to integrate the techniques of behavioural activation, the vertical arrow technique and Socratic questioning to change negative thoughts and identify positive attitudes into their repertoire of skills and implement them according to the patient's needs.

It is clear that there is a need for further studies on treatment integrity in the cognitive therapy of depression. Future studies might look at the development of a standard conceptualisation of treatment integrity, the effect of specific techniques such as the challenging of maladaptive thoughts, the utility of thought in behavioural activation and the establishment of competent training in cognitive therapy.

\section{References}

American Psychiatric Association (2013) Diagnostic and Statistical Manual of Mental Disorders (5 $5^{\text {th }}$ edn) (DSM-5). American Psychiatric Publishing.

Arnow BA, Blasey C, Manber R, Constantino MJ, Markowitz JC, Klein DN, Thase ME, Kocsis JH, Rush AJ (2007) Dropouts versus completers among chronically depressed outpatients. Journal of Affective Disorders, 97(1-3): 197-202.

Bados A, Balaguer G, Saldaña C (2007) The efficacy of cognitive-behavioral therapy and the problem of drop-out. Journal of Clinical Psychology, 63: 585-92.

Bambling M, King R, Raue P, et al (2006) Clinical supervision: its influence on client-rated working alliance and client symptom reduction in the brief treatment of major depression. Psychotherapy Research, 16: 317-31.

Beck AT (1979) Cognitive Therapy of Depression. Guilford press.

Beck AT, Alford BA (2009) Depression: Causes and Treatment. University of Pennsylvania Press.

Beck AT, Davis DD, Freeman A (2015) Cognitive Therapy of Personality Disorders. Guilford Publications.

Beck SJ, Broder RF (2016) The new 'homework' in cognitive behaviour therapy (blog). Beck Institute for Cognitive Behavior Therapy (https:// www.beckinstitute.org/the-new-homework-in- cognitive-behavior-therapy). Accessed 24 November 2017

Bennett ST, Flett RA, Babbage DR (2014) Culturally adapted cognitive behaviour therapy for Māori with major depression. Cognitive Behaviour Therapist, 7: e20.

Bockting CL, Schene AH, Spinhoven P, et al (2005) Preventing relapse/ recurrence in recurrent depression with cognitive therapy: a randomized controlled trial. Journal of Consulting and Clinical Psychology, 73: 647-57.

Cuijpers P, Berking M, Andersson G, et al (2013) A meta-analysis of cognitive-behavioural therapy for adult depression, alone and in comparison with other treatments. Canadian Journal of Psychiatry, 58: 376-85.

DeRubeis RJ, Hollon SD, Amsterdam JD, et al (2008) Cognitive therapy vs medications in the treatment of moderate to severe depression. Archives of General Psychiatry, 62: 409-16.

Dimidjian S, Hollon SD, Dobson KS, et al (2006) Randomized trial of behavioral activation, cognitive therapy, and antidepressant medication in the acute treatment of adults with major depression. Journal of Consulting and Clinical Psychology, 74: 658-70.

Fava M, Alpert JE, Carmin CN, et al (2004) Clinical correlates and symptom patterns of anxious depression among patients with major depressive disorder in STAR*D. Psychological Medicine, 34: 1299-308.

Forand NR, DeRubeis RJ (2013) Pretreatment anxiety predicts patterns of change in cognitive behavioral therapy and medications for depression. Journal of Consulting and Clinical Psychology, 81: 774.

Haaga DA, Dyck MJ, Ernst D (1991) Empirical status of cognitive theory of depression. Psychological Bulletin, 110: 215-36.
MCO answers

1 c 2 e 3 a 4 a 5 e 
Hollon SD, DeRubeis RJ, Shelton RC, et al (2005) Prevention of relapse following cognitive therapy vs medications in moderate to severe depression. Archives of General Psychiatry, 62: 417-22.

Horrell SCV (2008) Effectiveness of cognitive-behavioral therapy with adult ethnic minority clients: a review. Professional Psychology: Research and Practice, 39: 160-8.

Jacobson NS, Dobson KS, Truax PA, et al (1996) A component analysis of cognitive-behavioral treatment for depression. Journal of Consulting and Clinical Psychology, 64: 295-304.

Johnsen TJ, Friborg 0 (2015) The effects of cognitive behavioral therapy as an anti-depressive treatment is falling: a meta-analysis. Psychological Bulletin, 141: 747-68.

Kaslow NJ, Borden KA, Collins FL, et al (2004) Competencies conference: future directions in education and credentialing in professional psychology. Journal of Clinical Psychology, 60: 699-712.

Kazantzis N, Deane FP, Ronan KR (2000) Homework assignments in cog nitive and behavioral therapy: a meta-analysis. Clinical Psychology: Science and Practice, 7: 189-202.

Keefe JR, Webb CA, DeRubeis RJ (2016) In cognitive therapy for depression, early focus on maladaptive beliefs may be especially efficacious for patients with personality disorders. Journal of Consulting and Clinical Psychology, 84: 353-64.

Lambert MJ, Hansen NB, Finch AE (2001) Patient-focused research: using patient outcome data to enhance treatment effects. Journal of Consulting and Clinical Psychology, 69: 159-72.

Longmore RJ, Worrell M (2007) Do we need to challenge thoughts in cognitive behavior therapy? Clinical Psychology Review, 27: 173-87.

Ma SH, Teasdale JD (2004) Mindfulness-based cognitive therapy for depression: replication and exploration of differential relapse prevention effects. Journal of Consulting and Clinical Psychology, 72: 31-40.

Martell CR, Addis ME, Jacobson NS (2001) Depression in Context: Strategies for Guided Action. WW Norton.

McGlinchey JB, Dobson KS (2003) Treatment integrity concerns in cognitive therapy for depression. Journal of Cognitive Psychotherapy, 17: 299318.

Naeem F, Gul M, Irfan M, et al (2015) Brief culturally adapted CBT (CaCBT) for depression: a randomized controlled trial from Pakistan. Journal of Affective Disorders, 177: 101-7.

National Institute for Health and Care Excellence (2009) Depression in Adults: Recognition and Management (NICE Clinical Guideline CG90). NICE.

Newton-Howes G, Tyrer P, Johnson T, et al (2014) Influence of personality on the outcome of treatment in depression: systematic review and metaanalysis. Journal of Personality Disorders, 28: 577-93.

Pentaraki DA (2012) Breaking the Chains of Women's Depression. Brain Matters Institute.
Rees CS, McEvoy P, Nathan PR (2005) Relationship between homework completion and outcome in cognitive behaviour therapy. Cognitive Behaviour Therapy, 34: 242-7.

Richards DA, Ekers D, McMillan D, et al (2016) Cost and Outcome of Behavioural Activation versus Cognitive Behavioural Therapy for Depression (COBRA): a randomised, controlled, non- inferiority trial. Lancet, 388: 871-80.

Roth $A D$, Pilling $S$ (2008) Using an evidence-based methodology to identify the competences required to deliver effective cognitive and behavioural therapy for depression and anxiety disorders. Behavioural and Cognitive Psychotherapy, 36: 129-47.

Shaw BF, Elkin I, Yamaguchi J, et al (1999) Therapist competence ratings in relation to clinical outcome in cognitive therapy of depression. Journal of Consulting and Clinical Psychology, 67: 837-46.

Simpson-Southward C. Waller G. Hardy GE (2016) Supervision for treatment of depression: an experimental study of the role of therapist gender and anxiety. Behaviour Research and Therapy, 77: 17-22.

Swift JK, Greenberg RP (2014) A treatment by disorder meta-analysis of dropout from psychotherapy. Journal of Psychotherapy Integration, 24: 193.

Teasdale JD, Segal ZV, Williams JMG, et al (2000) Reducing risk of recurrence of major depression using Mindfulness-Based Cognitive Therapy. Journal of Consulting \& Clinical Psychology, 68: 615-23.

Trepka C, Rees A, Shapiro DA, et al (2004) Therapist competence and outcome of cognitive therapy for depression. Cognitive Therapy and Research, 28: 143-57.

Uher R, Payne JL, Pavlova B, et al (2014) Major depressive disorder in DSM-5: implications for clinical practice and research of changes from DSM-IV. Depression and Anxiety, 31: 459-71.

Veale D (2008) Behavioural activation for depression. Advances in Psychiatric Treatment, 14: 29-36.

Waltman SH, Creed TA, Beck AT (2016) Are the effects of cognitive behavior therapy for depression falling? Review and critique of the evidence. Clinical Psychology: Science and Practice, 23: 113-22.

Waltz J, Addis ME, Koerner K, et al (1993) Testing the integrity of a psychotherapy protocol: assessment of adherence and competence. Journal of Consulting and Clinical Psychology, 61: 620.

Westbrook D, Kennerley H, Kirk J (2007) An Introduction to Cognitive Behaviour Therapy: Skills and Applications. Sage Publications.

White E, Winstanley J (2010) A randomised controlled trial of clinical supervision: selected findings from a novel Australian attempt to establish the evidence base for causal relationships with quality of care and patient outcomes, as an informed contribution to mental health nursing practice development. Journal of Research in Nursing, 15: 151-67.

Wierzbicki M, Pekarik G (1993) A meta-analysis of psychotherapy dropout. Professional Psychology: Research and Practice, 24: 190-5. 
MCOs

Select the single best option for each question stem

1 Within cognitive therapy, depression is conceptualised as:

a an illness of the brain that results in cognitive dysfunction

b the result of faulty thinking and avoidance behaviour

c a 'depressive cognitive triad', characterised by a negative maladaptive thought pattern about oneself, the world and the future

d low motivation, negative thinking and inability to sleep

e difficulty in concentrating, a maladaptive thought pattern and memory deficits.

2 Drop-out rates in cognitive therapy for affective disorders have been associated with patients':

a faulty thinking, low motivation and difficulty in concentrating

b unemployment, low motivation and negative thinking c low motivation, lack of volition and inability to concentrate

d low motivation, transportation problems and dissatisfaction with the therapist

e low motivation, dissatisfaction with the therapist/treatment and external factors.

3 Regarding treatment integrity in cognitive therapy for depression:

a pragmatic aspects of cognitive therapy such as the establishment of a session's agenda and homework assignments positively correlate with patients' improvement

b no studies have examined treatment integrity in cognitive therapy for depression

c the therapist's exploration of patient's underlying assumptions and personal meanings correlates with the clinical outcome

d treatment integrity relates to behavioural activation and homework assignments

e the patient's motivation is of paramount importance.
4 Action plans (homework) in the form of behavioural tasks (exposure and pleasurable activities) are more effective for the reduction of:

a depression

b anxiety and depression

c panic attacks

d somatic symptoms

e negative behaviour.

5 Specific cognitive therapy techniques that are associated with an effective clinical outcome in depression involve:

a CT-concrete variables, the patient's motivation and good action plans

b the development of a good therapeutic relationship

c action plans that increase the patient's motivation to engage in therapy

d Socratic questioning, behavioural activation and generic therapeutic skills

e CT-concrete variables, action plans, generic therapeutic skills, expertise in cognitive therapy techniques and behavioural activation. 